\title{
Status of the CALET Ultra Heavy Cosmic Ray Analysis
}

\author{
Brian Flint Rauch*i \\ Department of Physics and McDonnell Center for the Space Sciences, Washington University, \\ St. Louis, MO 63130 USA \\ E-mail: brauchephysics.wust1.edu
}

\author{
Yosui Akaike \\ University of Maryland Baltimore County and NASA Goddard Space Flight Center, Greenbelt, \\ MD 20771, USA
}

E-mail: yosui.akaikednasa.gov

\section{for the CALET Collaboration}

\begin{abstract}
The CALorimetric Electron Telescope (CALET) was launched to the International Space Station (ISS) on August 19, 2015, and has been returning science data since October 13, 2015. Through the main calorimeter (CAL), CALET observes the fluxes of high-energy electrons, gamma rays and nuclei. CALET measures the energy spectra of the more abundant cosmic-ray nuclei through ${ }_{26} \mathrm{Fe}$ passing within the full CAL geometry, and utilizing an ultra-heavy cosmic-ray (UHCR) trigger, measures the relative abundances of the rare UHCR nuclei through ${ }_{40} \mathrm{Zr}$ with an expanded geometric acceptance. Preliminary analysis of the ${ }_{26} \mathrm{Fe}$ statistics from the first $\sim 13$ months of CAL data passing the UHCR trigger have validated the preflight estimate that in a 5 year mission CALET will observe comparable UHCR statistics to those achieved in the first flight of the SuperTIGER balloon-borne UH experiment. The CALET UHCR measurements will complement those by SuperTIGER in a similar energy range without the need to correct for atmospheric interactions, as well as those at lower energy and with lower statistics by the space-based ACE-CRIS instrument. CALET is unique as an instrument sensitive to UHCR in having the dynamic range to measure from ${ }_{1} \mathrm{H}$ to ${ }_{40} \mathrm{Zr}$. We present the status of the CALET UHCR analysis.
\end{abstract}

35th International Cosmic Ray Conference - ICRC2017-

10-20 July, 2017

Bexco, Busan, Korea

\footnotetext{
${ }^{*}$ Speaker.

${ }^{\dagger}$ CALET supported by JAXA in Japan, ASI in Italy and in the USA by NASA grant \#NNX11AE02G.
} 


\section{Introduction}

The CALorimetric Electron Telescope (CALET) is a Japanese-Italian-US astroparticle observatory on the International Space Station (ISS) [U]. CALET was launched on August 19, 2015 on JAXA's HTV-5, and it began science operations on October 13, 2015. As the name implies, the primary science objective of CALET is to measure the fluxes of the highest energy cosmic-ray electrons $(1 \mathrm{GeV}$ to $20 \mathrm{TeV}$ ) [Q], B], but the instrument also observes gamma rays ( $10 \mathrm{GeV}$ to 10 $\mathrm{TeV}$ ) [四, []] and cosmic-ray nuclei (up to $1,000 \mathrm{TeV}$ ) [ [6, 四]. These measurements are made using the main calorimeter (CAL), which is shown in the CALET instrument package in the left of Fig. $\mathrm{W}$. Other CALET detector systems are also shown, including the CALET Gamma Ray Burst Monitor (CGBM) [8]].
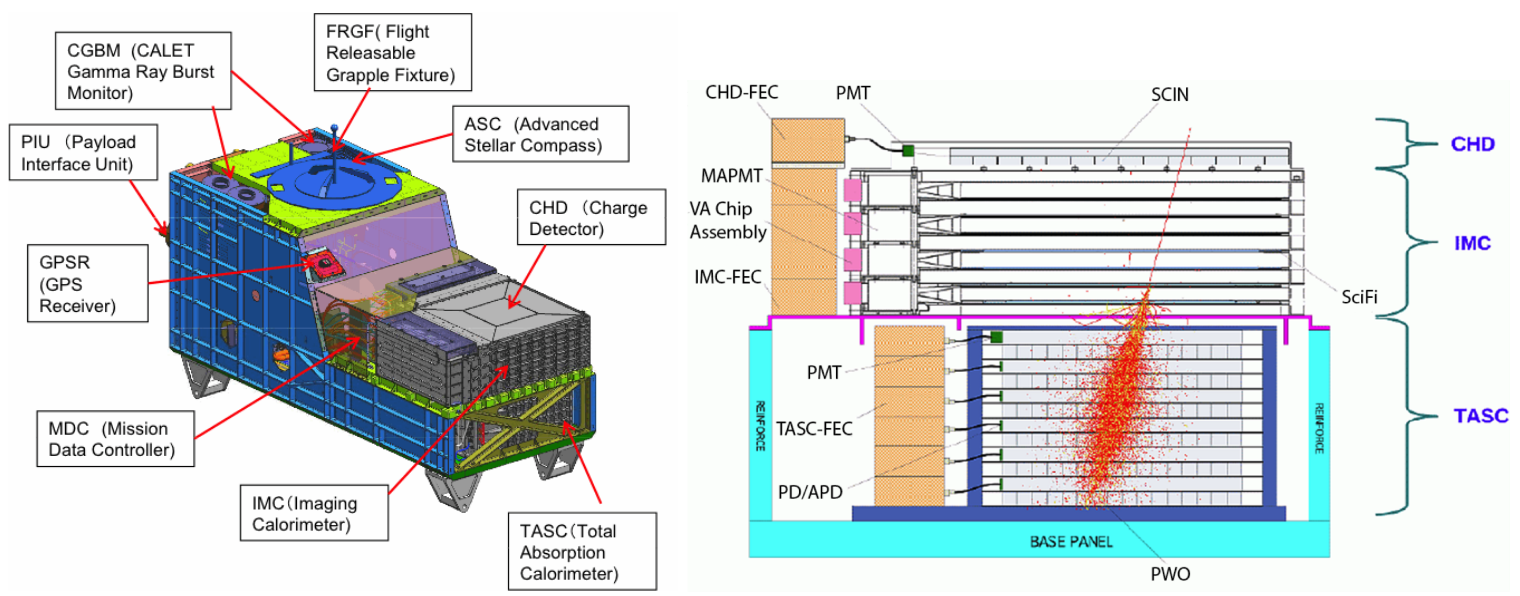

Figure 1: Left: CALET instrument package showing CAL and CGBM subsystems. Right: CAL side-view showing CHD, IMC, and TASC detectors with an example image of a simulated $1 \mathrm{TeV}$ electron shower.

The CAL, shown from the side in the right of Fig. I, consists of three stacked detector systems. At the top of the CAL is the CHarge Detector module (CHD) comprised of $\mathrm{x}$ - and y-layers of 14 crossed $32 \mathrm{~mm}$ wide by $10 \mathrm{~mm}$ thick by $450 \mathrm{~mm}$ long scintillator paddles. In the middle is a 156.5 $\mathrm{mm}$ tall IMaging Calorimeter (IMC) made up of $8 \mathrm{x}$ - and y-layers of $1 \mathrm{~mm}$ square $448 \mathrm{~mm}$ long scintillating fibers. The fiber layers are interspersed with 3.0 radiation lengths $\left(X_{\circ}\right)$ of tungsten: 5 that are $0.2 X_{\circ}$ thick above 2 layers that are $1.0 X_{\circ}$ thick. At the bottom is the Total AbSorption Calorimeter (TASC) composed of $6 \mathrm{x}$ - and y-layers of $16 \mathrm{PWO}$ logs that are $19 \mathrm{~mm}$ wide, $20 \mathrm{~mm}$ tall and $326 \mathrm{~mm}$ long. The combined calorimeter depth of the CAL is $30 X_{\circ}$, with $3 X_{\circ}$ in the IMC and $27 X_{\circ}$ in the TASC.

\section{Ultra-Heavy Cosmic Rays}

The ultra-heavy cosmic rays (UHCR) comprised of the nuclei with charge greater than or equal to ${ }_{30} \mathrm{Zn}$ are rare and provide clues about cosmic-ray origins. Figure $\square$ is a plot of the relative abundances of the cosmic rays at $2 \mathrm{GeV} /$ nuc compared with those for the Solar System $\left({ }_{14} \mathrm{Si}=1\right)$. We see that the UHCR with charges $30 \leq Z \leq 40$ are $\sim 10^{5}$ times less abundant than ${ }_{26} \mathrm{Fe}$, which in turn is $\sim 5 \times 10^{3}$ times less abundant than ${ }_{1} \mathrm{H}$. The UHCR have so far only been measured 
with single element precision up to ${ }_{40} \mathrm{Zr}$ by the TIGER [Q] and SuperTIGER [0] balloon-borne instruments at GeV/nuc energies, and by the ACE-CRIS [W] space based instrument at hundreds of $\mathrm{MeV} /$ nuc. These measurements of the elemental composition of the UHCR show enhancements that can be explained by contributions of source material from massive star outflows and supernova ejecta mixed in with normal interstellar medium (ISM). This suggests that a significant fraction of the cosmic rays originate in OB Associations where most massive stars reside and where the majority of the supernovae, generally believed to accelerate the Galactic cosmic rays (GCR), occur. Further, UHCR and lower mass cosmic ray source abundances indicate that there is an atomic mass $(A)$ dependence in the acceleration of the GCR, as well as a significant enhancement in the abundance of the refractory elements that form dust grains over the volatile elements that exist as gas.

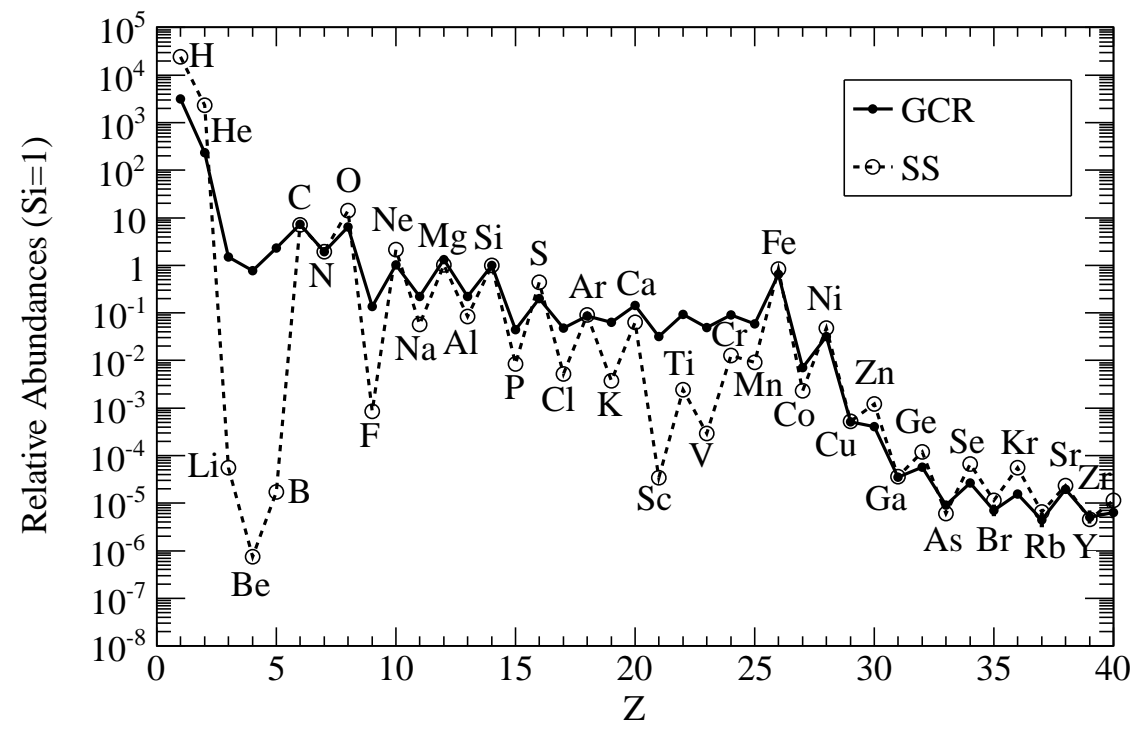

Figure 2: Solar System (SS) [[2] and Galactic cosmic-ray (GCR) relative abundances at $2 \mathrm{GeV} / \mathrm{nuc}(1 \leq$

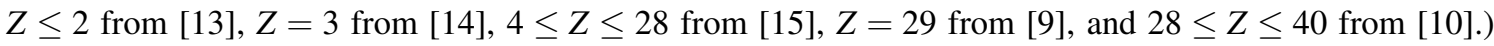
normalized to ${ }_{14} \mathrm{Si}=1$.

CALET has the capability to make a measurement of the UHCR abundances that will complement the existing data set and add significantly to its overall statistics. The CHD has the dynamic range to measure nuclei with charges just beyond ${ }_{40} \mathrm{Zr}$. The ISS is in a $51.6^{\circ}$ inclination orbit and the geomagnetic field limits the low-energy cosmic-ray flux so that CALET sees a similar UHCR energy range to TIGER/SuperTIGER. With the anticipated five year exposure on the ISS CALET is expected to collect comparable UHCR statistics to those obtained in the first SuperTIGER flight [ㅁ] ]. In addition, the ability of CALET to measure cosmic-ray abundances in the $1 \leq Z \leq 40$ charge range with a single instrument will provide a valuable check of cross calibrations for these other measurements. Preliminary analysis results for measurements of nuclei up to the UHCR range [6, 四] demonstrate that CALET has this capability. 


\section{Analysis Method}

\subsection{Initial CALET Data Processing}

CALET data volumes undergo significant processing in preparation for use in specific data analysis efforts. The data are received at the Waseda CALET Operations Center (WCOC), which monitors and controls CALET and prepares the data volumes for distribution to the international collaboration [ए]]. In processing the data for use in other analysis efforts a number of calibrations are applied to correct for variations in instrument response over time and as a function of position within detector elements. These corrections are continuously updated by using minimum-ionizing particles (MIPs) of cosmic-ray hydrogen and helium nuclei passing through the instrument [1], [1]]. At this stage, primary analysis products are also provided, including the calibrated energy scale [20] and event track reconstructions [제]. The processed data volumes are then made available for distribution to the rest of the collaboration.
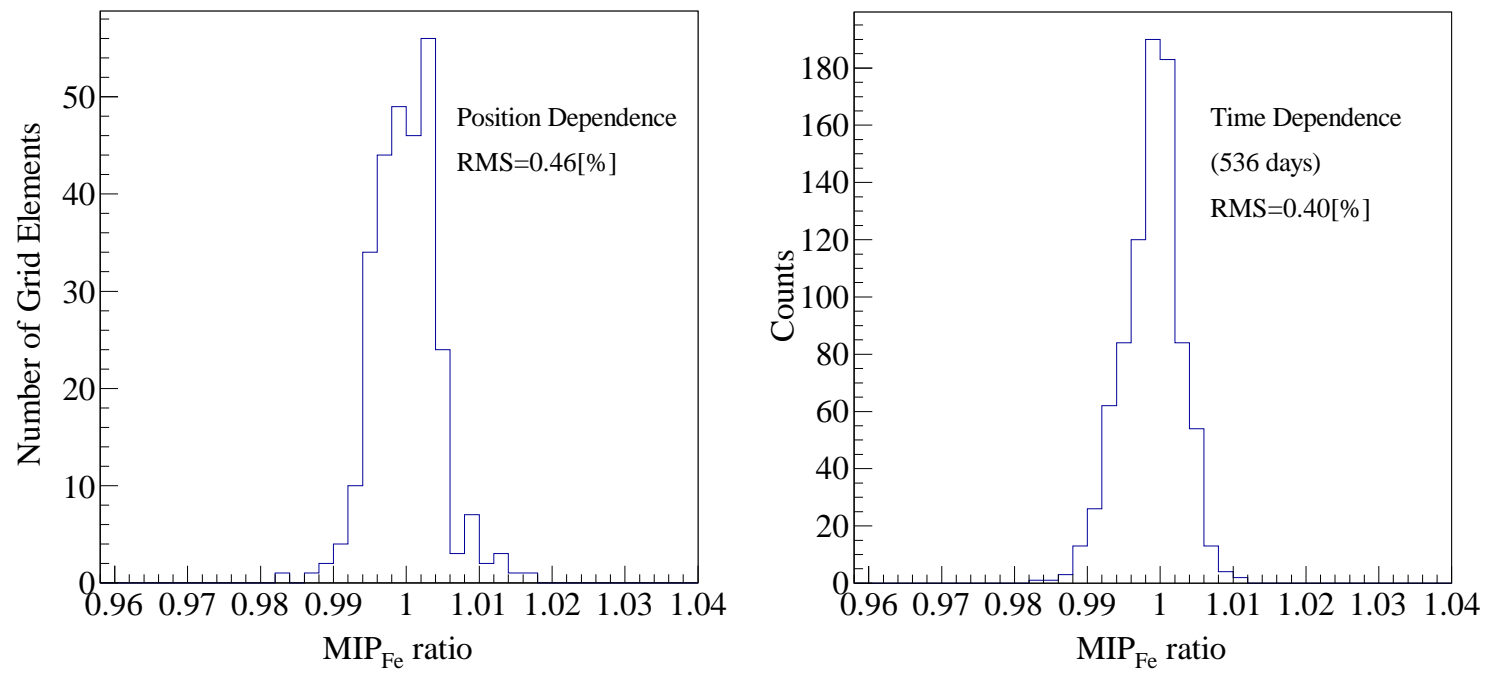

Figure 3: Left: Variation in ${ }_{26} \mathrm{Fe}$ peak signal with position in the CHD in the preliminary UHCR analysis. Right: Variation in ${ }_{26} \mathrm{Fe}$ peak signal with time in CHD in the preliminary UHCR analysis.

\subsection{UHCR Data Analysis}

As is the case in other analysis efforts, the CALET UHCR analysis involves its own specific data corrections and cuts. Data for this analysis are selected from events that had an UH trigger (detailed below) and for which a track could be reconstructed. The primary instrument response corrections made with protons and helium are good to approximately $1 \%$ for light nuclei. For the UHCR analysis the charge resolution can be improved by applying corrections to CHD calibrated signals based on variations in the ${ }_{26} \mathrm{Fe}$ peak over time and position within the detector. The plot on the left in Fig. [3] shows the residual variations in the ${ }_{26} \mathrm{Fe}$ peak signals in the CHD with position in the detector after ${ }_{26} \mathrm{Fe}$ based corrections were applied for the 14 by $14 \mathrm{CHD}$ paddle grid. The geometric acceptance used in this preliminary analysis excluded events within $3.2 \mathrm{~cm}$ of the edge of the CHD for $\sim 73 \%$ of the potential UH trigger geometry. The right plot in Fig. [3] similarly shows 
the residual variations in the ${ }_{26} \mathrm{Fe}$ peak signals with time after ${ }_{26} \mathrm{Fe}$ based corrections were applied. The CHD signal corrections for such nonuniform responses are needed to achieve the $\sim 0.5 \%$ signal resolution required to resolve the UHCR. A charge consistency cut requiring agreement between CHDX and CHDY to within $15 \%$ is applied, as in [ $[\mathbf{D}]$. Finally, a charge assignment is made accounting for scintillator saturation following the method in [22].

\subsection{Geomagnetic Energy Threshold Cut}

The CALET UHCR analysis will utilize geomagnetic energy threshold cuts to maximize the geometric acceptance for UHCR [ए]]. This scheme utilizes a UH trigger requiring above threshold signals in the CHD and top four IMC layers, which has a significantly larger geometry factor $(0.44$

$\left.\mathrm{m}^{2} \mathrm{sr}\right)$ than for events contained by the TASC $\left(0.07 \mathrm{~m}^{2} \mathrm{sr}\right)$, but at the cost of foregoing TASC energy measurement. The concept is that sufficient UHCR charge resolution can be achieved with an energy threshold cut based on geomagnetic rigidity that eliminates lower energy events where the scintillation signal varies most strongly with energy.
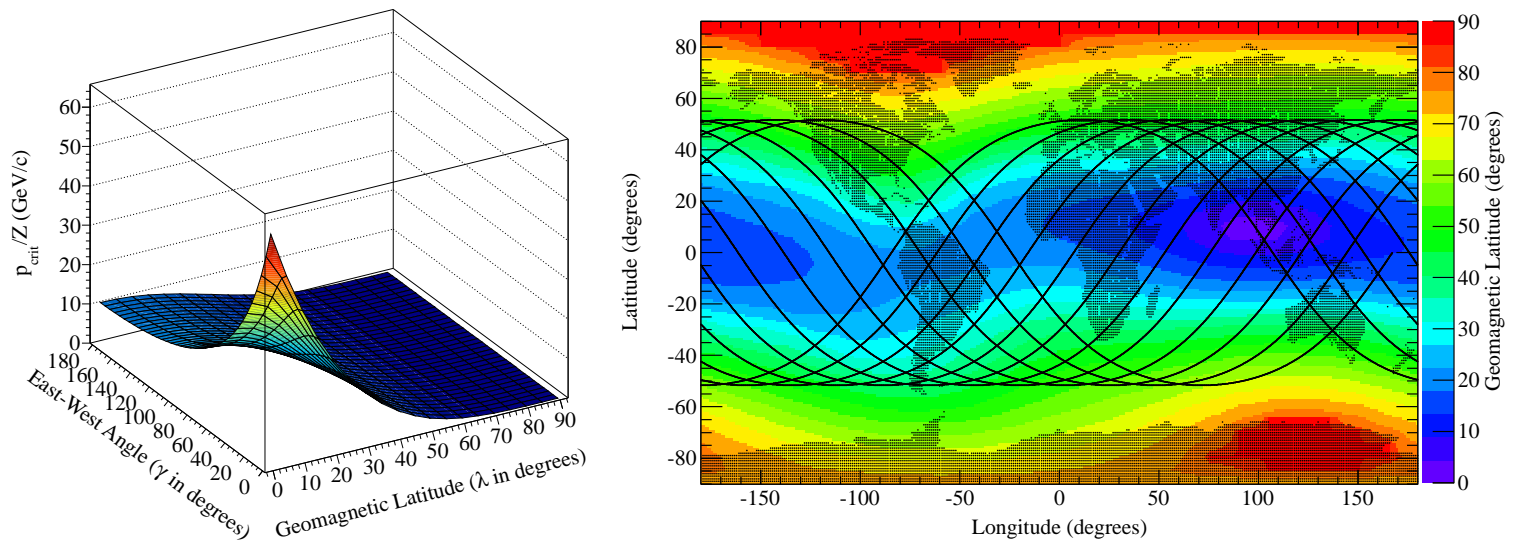

Figure 4: Left: Cutoff rigidity (critical momentum per charge) [23]. Right: Contour plot of geomagnetic latitude at $450 \mathrm{~km}$ in $1^{\circ}$ longitude and latitude bins derived from [24]. ISS orbit of $51.6^{\circ}$ inclination is shown in black curves.

The threshold required for charged particles to penetrate the geomagnetic field depends on their trajectories relative to the field direction. This is determined by the cutoff rigidity, which is the critical momentum, $p_{\text {crit }}(\gamma, \lambda)$ (Eq. B.D) [23], divided by the atomic number, $R_{\text {cutoff }}=p_{\text {crit }}(\gamma, \lambda) / Z$. The left plot in Fig. $\mathbb{t}$ shows $R_{\text {cutoff }}$ as a function of geomagnetic latitude $(\lambda)$ and the transverse East-West angle relative to the field lines $(\gamma)$. The minimum kinetic energy per AMU required for a particle to penetrate the geomagnetic field is given by Eq. B.2, which shows that the energy threshold $E_{c r i t}$ depends on the ratio of critical momentum to atomic mass, and so (at a fixed cutoff rigidity) by the ratio of atomic number to atomic mass. The geomagnetic latitudes for $p_{c r i t}(\gamma, \lambda)$ and $E_{c r i t}$ are shown for the ISS orbit at $\sim 400 \mathrm{~km}$ on the right in Fig. $\rightarrow$. These were derived from the geomagnetic vertical cutoff rigidities at an altitude of $450 \mathrm{~km}$ [D4] using $R_{\text {cut }}=15 \cos ^{4}(\lambda) \mathrm{GV}$ [23] after interpolating the vertical cutoff rigidities to a $1^{\circ}$ pitch. 


$$
\begin{aligned}
p_{\text {crit }}(\gamma, \lambda) & =60 Z\left[\frac{1-\sqrt{1-\cos (\gamma) \cos ^{3}(\lambda)}}{\cos (\gamma) \cos (\lambda)}\right]^{2} \\
E_{c r i t} & =\sqrt{p_{c r i t}^{2} / A^{2}+m_{\text {amu }}^{2}}-m_{\text {amu }}
\end{aligned}
$$

The full geomagnetic rigidity cut with the East-West angle dependence has not yet been implemented in this preliminary CALET UHCR analysis. The event specific critical energies can be determined from the trajectory and geospatial information that are available in the CALET data, and including such a threshold cut is the next major analysis step. The model predictions that this method will enable CALET to observe comparable UHCR statistics to the first flight of SuperTIGER [16] have been validated by ${ }_{26} \mathrm{Fe}$ statistics from preliminary analysis of the first $\sim 13$ months of CAL data passing the UHCR trigger agreeing with those predicted by the model to within $10 \%$.

\section{Preliminary Results}

The results of this preliminary CALET UHCR analysis for the first 18 months of CALET data are shown in Fig. [. This charge histogram shown on the left in Fig. \ is for events that passed the CALET UH trigger, had reconstructed trajectories, were within $60^{\circ}$ of vertical, passed the CHD charge consistency cuts, and which had a geomagnetic vertical cutoff rigidity $\geq 4 \mathrm{GV}$. Using the vertical rigidity cut on such a wide incidence-angle acceptance leaves some spillover from the more abundant lower- $Z$ peaks that obscure ${ }_{30} \mathrm{Zn}$ and ${ }_{31} \mathrm{Ga}$ as shoulders, but one sees peaks for ${ }_{32} \mathrm{Ge},{ }_{34} \mathrm{Se}$, ${ }_{36} \mathrm{Kr}$ and ${ }_{38} \mathrm{Sr}$. The relatively less-abundant odd $-Z$ are not as clearly resolved and are proportionally more contaminated by the tails of their adjacent lower charge even- $Z$ peaks.
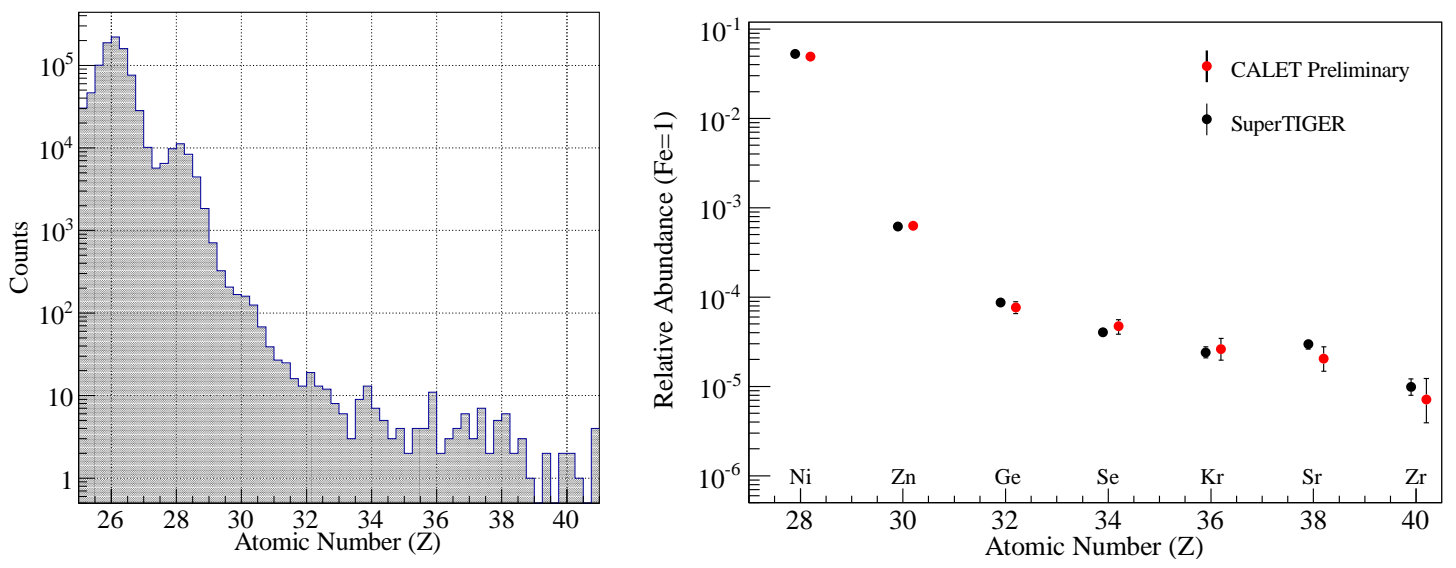

Figure 5: Left: Preliminary CALET UHCR charge histogram with 4 bins/charge unit for UH trigger events within $60^{\circ}$ of vertical and for a geomagnetic vertical cutoff rigidity $>4 \mathrm{GV}$. Right: Even- $Z$ relative abundances $\left({ }_{26} \mathrm{Fe}=1\right)$ for CALET (red points) compared with SuperTIGER (black points) [ए]].

The right plot of Fig. [ 5 shows that the relative UHCR abundances of the even- $Z$ elements from this preliminary CALET analysis agree with those from SuperTIGER [ए]] within statistical 
uncertainties. The abundance data were derived by summing events within \pm 0.5 charge units (cu) of each element charge and deducting the background from Gaussian fits to the adjacent peaks. The background was determined from a multiple-Gaussian function assuming a constant standard deviation of $0.35 \mathrm{cu}$ (the standard deviation of the ${ }_{26} \mathrm{Fe}$ peak in this analysis) and that the peaks are centered on integral charges. This analysis also found that the CALET relative abundances for the even-Z UHCR were consistent within statistical uncertainties for vertical rigidity cuts of 3, 4 and $5 \mathrm{GV}$, but that the relative abundances below ${ }_{34} \mathrm{Se}$ were elevated for a $2 \mathrm{GV}$ cut, likely due to tails on the ${ }_{26} \mathrm{Fe}$ and ${ }_{28} \mathrm{Ni}$ distributions. The odd- $Z$ UHCR elements are not shown as they were found to have elevated relative abundances compared to those from SuperTIGER owing to contamination from the tails of more abundant adjacent peaks. We expect that further analysis will reduce the low-energy spillover from lower charges and allow us to resolve the odd- $Z$ abundances as well.

\section{Conclusions}

CALET has the capability to measure the relative abundances of the UHCR. Refining the analysis to include a more detailed geomagnetic threshold cut based on particle trajectories relative to the geomagnetic field is expected to provide good charge resolution over the increased geometric acceptance available from the UH trigger. In five years of observation CALET will be able to make space-based UHCR measurements at comparable energies and with similar statistics to what was achieved with the first SuperTIGER flight with the advantage of not requiring corrections for atmospheric interactions. Further, by measuring the cosmic ray charge spectrum from $1 \leq Z \leq 40$ with a single instrument CALET will provide cross calibrations for other measurements.

\section{References}

[1] S. Torii for the CALET Collaboration, The CALorimetric Electron Telescope (CALET) on the ISS: Preliminary Results from the On-orbit Observation since October, 2015, in proceedings of The 35th International Cosmic Ray Conference, POS (ICRC2017) (2017).

[2] Y. Asaoka for the CALET Collaboration, Analysis and Preliminary Results for the Cosmic Ray Electron Spectrum from CALET, in proceedings of The 35th International Cosmic Ray Conference, POS (ICRC2017) 205 (2017).

[3] L. Pacini and Y. Akaike for the CALET Collaboration, Capability of electron identification for the CALET measurement, in proceedings of The 35th International Cosmic Ray Conference, $\mathrm{DOS}$ (ICRC2O17) 163 (2017).

[4] N. Cannady for the CALET Collaboration, High-Energy Gamma-ray Observations Using the CALorimetric Electron Telescope, in proceedings of The 35th International Cosmic Ray Conference, POS (ICRC2017) T20 (2017).

[5] M. Mori for the CALET Collaboration, Search for gamma-ray emission from electromagnetic counterparts of gravitational wave sources with the CALET calorimeter, in proceedings of The 35th International Cosmic Ray Conference, POS (ICRC2017) 637 (2017).

[6] P.S. Marrocchesi for the CALET Collaboration, Observation of Protons and Light Nuclei with CALET: Analysis and Preliminary Results, in proceedings of The 35th International Cosmic Ray Conference, POS (ICRC2017) 156 (2017). 
[7] Y. Akaike for the CALET Collaboration, Measurements of heavy nuclei with the CALET experiment, in proceedings of The 35th International Cosmic Ray Conference, POS (ICRC2017) 181 (2017).

[8] K. Yamaoka for the CALET Collaboraiton, CALET GBM Observations of Gamma-ray Bursts and Gravitational Wave Sources, in proceedings of The 35th International Cosmic Ray Conference, एतS (ICRC2017) 614 (2017).

[9] B.F. Rauch et al., Cosmic Ray origin in OB Associations and Preferential Acceleration of Refractory Elements: Evidence from Abundances of Elements ${ }_{26} \mathrm{Fe}$ through ${ }_{34} \mathrm{Se}, \mathrm{ApJ}, 697$ (2009) 2083-2088, [arXiv:0906.2021].

[10] R.P. Murphy et al., Galactic Cosmic Ray Origins and OB Associations: Evidence from SuperTIGER Observations of Elements ${ }_{26} \mathrm{Fe}$ Through ${ }_{40} \mathrm{Zr}, \mathrm{ApJ}, \mathbf{8 3 1}$ (2016) 2083-2088, [arXiv: 1608 . 08183].

[11] W.R. Binns et al., Constraints on Galactic Cosmic-Ray Origins from Elemental and Isotopic Composition Measurements, in proceedings of The 33rd International Cosmic Ray Conference (Rio de Janeiro), (2013), paper 0646, http://www.cbpf.br/ icrc2013/papers/icrc2013-0646.pdf.

[12] K. Lodders, Solar System Abundances and Condensation Temperatures of the Elements, ApJ, 591 (2003) 1220-1247.

[13] T. Sanuki et al., Precise Measurement of Cosmic-Ray Proton and Helium Spectra with the BESS Spectrometer, ApJ, 545 (2000) 148-155, [arXiv: astro-ph/ 0002481 ].

[14] M. Aguilar et al., Isotopic Composition of Light Nuclei in Cosmic Rays: Results from AMS-01, ApJ, 736 (2011) 105-116, [arXiv: 1106.2269$].$

[15] J.J. Engelmann et al., Charge composition and energy spectra of cosmic-ray nuclei for elements from Be to NI - Results from HEAO-3-C2, A\&A, 233 (1990) 96-111.

[16] B.F. Rauch for the CALET Collaboration, Predicted CALET Measurements of Heavy and Ultra-Heavy Cosmic Ray Nuclei, in proceedings of The 34th International Cosmic Ray Conference, POS (ICRC2015) 627 (2015).

[17] S. Ozawa and Y. Asaoka for the CALET Collaboration, CALET on-orbit operations and data analysis system at the Waseda CALET Operations Center (WCOC), in proceedings of The 35th International Cosmic Ray Conference, POS (ICRC2017) 165 (2017).

[18] Y. Asaoka et al., Energy calibration of CALET onboard the International Space Station, APh, 91 (2017) 1-10.

[19] Y. Komiya and G. Bigongiari for the CALET Collaboration, MIP Calibration and the Long-term Stability of CALET onboard the International Space Station, in proceedings of The 35th International Cosmic Ray Conference, POS (ICRC2017) 206 (2017).

[20] R. Miyata and the CALET Collaboration, Full Dynamic Range Energy Calibration of CALET onboard the International Space Station, in proceedings of The 35th International Cosmic Ray Conference, POS (ICRC2017) 207 (2017).

[21] P. Maestro and N. Mori for the CALET Collaboration, Particle tracking in the CALET experiment, in proceedings of The 35th International Cosmic Ray Conference, POS (ICRC2017) 208 (2017).

[22] P.S. Marrocchesi et al., Beam test performance of a scintillator-based detector for the charge identification of relativistic ions, NIMPA, 659 (2011) 477-483.

[23] C. Störmer, The Polar Aurora, Oxford University, London 1950.

[24] D.F. Smart \& M.A. Shea, Fifty years of progress in geomagnetic cutoff rigidity determinations, AdSpR, 44 (2009) 1107-1123. 